\title{
Analysis of Type-II Censored Competing Risks' Data under Reduced New Modified Weibull Distribution
}

\author{
Saad J. Almalki, ${ }^{1}$ Tahani A. Abushal ${ }^{(D},{ }^{2}$ M. D. Alsulami, ${ }^{3}$ and G. A. Abd-Elmougod ${ }^{4}{ }^{4}$ \\ ${ }^{1}$ Mathematics Department, Faculty of Science, Taif University, Taif, Saudi Arabia \\ ${ }^{2}$ Mathematics Department, Faculty of Science, Umm AL-Qura University, Mecca, Saudi Arabia \\ ${ }^{3}$ Mathematics Department, College of Sciences and Arts at Alkamil, University of Jeddah, Jeddah, Saudi Arabia \\ ${ }^{4}$ Mathematics Department, Faculty of Science, Damanhour University, Abadiyyat Damanhur, Egypt \\ Correspondence should be addressed to G. A. Abd-Elmougod; gam_amin@yahoo.com
}

Received 15 March 2021; Revised 6 April 2021; Accepted 21 April 2021; Published 29 May 2021

Academic Editor: Ahmed Mostafa Khalil

Copyright (c) 2021 Saad J. Almalki et al. This is an open access article distributed under the Creative Commons Attribution License, which permits unrestricted use, distribution, and reproduction in any medium, provided the original work is properly cited.

\begin{abstract}
Models with the bathtub-shaped hazard rate function are widely used in lifetime analysis and reliability engineering. In this paper, we adopted the reduced new modified Weibull (RNMW) distribution with a bathtub-shaped hazard rate function. Under consideration that the population units are failing with two independent causes of failure and the failure time is distributed with RNMW distribution, we formulate the model which is known as competing risks model. The model parameters under the type-II censoring scheme are estimated with the maximum likelihood method with the corresponding asymptotic confidence intervals. Also, the Bayes point and credible intervals with the help of MCMC methods are constructed. The real and simulated datasets are analyzed for illustrative purposes. Finally, the estimators are compared with the Monte Carlo simulation study.
\end{abstract}

\section{Introduction}

In the past few years, different modifications of Weibull distribution were constructed with a bathtub-shaped hazard rate function. These modifications were discussed with different authors, see [1-4]. Some modification models of Weibull distribution are discussed with with two or three parameters' vectors [4-6]. Also, some of the modifications of Weibull distribution were discussed with four parameters (see [7]) and the modifications with five parameters (see [8]). Then, the large sets of distribution are generalized with generalized linear exponential distribution as the bathtubshaped hazard rate presented by Sarhan et al. [9]. The unimodal, decreasing, increasing, or bathtub-shaped hazard rate beta-Weibull distribution was proposed by Lee et al. [10]. The four parameters' generalized modified Weibull distribution was proposed by Carrasco et al. [11].

Almalki and Yuan [12] have presented new modified Weibull distribution with five parameters and distribution function $(\mathrm{CDF})$ given by

$$
F(t)=1-e^{\left\{-\alpha_{1} \beta_{1}-\alpha_{2} t^{\beta_{2}} e^{\lambda t}\right\}}, \quad t>0, \alpha_{1}, \alpha_{2}, \beta_{1}, \beta_{2}, \lambda>0,
$$

where $\alpha_{1}$ and $\alpha_{2}$ are called shape parameters, $\beta_{1}$ and $\beta_{2}$ are called scale parameters, and $\lambda$ is called the accelerated parameter. Most effective and flexible lifetime distributions with bathtub-shaped hazard functions have more than three or four parameters but the reduced lifetime distributions have a few number of parameters, two or three, but these distributions are not much (for example, the modified Weibull distribution, the exponentiated Weibull distribution, and the modified Weibull extension). Almalki [13] presented a new version of new modified Weibull distribution with a bathtub-shaped failure rate function (FRF) called reduced new modified Weibull distribution by taking $\beta_{1}=\beta_{2}=(1 / 2)$, with CDF given by

$$
F(t)=1-e^{-\sqrt{t}\left\{\alpha_{1}+\alpha_{2} e^{\lambda t}\right\}}, \quad t>0, \alpha_{1}, \alpha_{2}, \lambda>0,
$$


where $\alpha_{1}$ and $\alpha_{2}$ are called scale parameters and $\lambda$ is called the accelerated parameter.

In this paper, we considered the special case of reduced version (2) called new reduced modified Weibull (NRMW) distribution with parameters $\alpha_{1}=\alpha_{2}=\alpha$ as follows:

$$
F(t)=1-e^{-\alpha \sqrt{t}\left\{1+e^{\lambda t}\right\}}, \quad t>0, \alpha, \lambda>0 .
$$

The density, survival, and hazard failure rate functions are given, respectively, by

$$
\begin{aligned}
f(t) & =\frac{\alpha}{2 \sqrt{t}}\left(1+(1+2 \lambda t) e^{\lambda t}\right) e^{-\alpha \sqrt{t}\left\{1+e^{\lambda t}\right\}}, \\
s(t) & =e^{-\alpha \sqrt{t}\left\{1+e^{\lambda t}\right\}}, \\
h(t) & =\frac{\alpha}{2 \sqrt{t}}\left(1+(1+2 \lambda t) e^{\lambda t}\right),
\end{aligned}
$$

where $\alpha$ and $\lambda$ are nonnegative parameters. The PDF of NRMW distribution has different shape, decreasing, or decreasing-increasing-decreasing, as shown in Figure 1. Also, different shapes of hazard failure rate function, decreasing, and the bathtub-shape are given in Figure 1.

If the random variable $T$ is distributed with NRMW, then the following two properties are satisfied.

(1) The $r$ th moment of the random variable of NRMW is given by

$$
\mu_{r}=2 r \sum_{i=0}^{\infty} \sum_{j=0}^{\infty} \frac{(-\alpha)^{i}(\lambda i)^{j}}{i ! j !} \frac{\Gamma(i+2(j+r))}{\alpha^{i+2(j+r)}},
$$

where $\Gamma(\cdot)$ is the gamma function.

(2) The moment generating function is given by

$$
M_{t}(\mathbf{t})=1+2 \sum_{i, j, k=0}^{\infty} \frac{(-\alpha)^{i}(i \lambda)^{j} t^{k+1}}{i ! j ! k !}\left[\frac{\Gamma(i+2(j+k)+2)}{\alpha^{i+2(j+k)+2}}\right] .
$$

The lifetime data of product units are presented in complete or censored data dependent on some consideration of the test time or cost. The word complete is used when the failure time data is obtained from all units under the test. But, the censored data is used when some but not all units under the test are failed through determined period of time. The censoring is available in various types; type-I and type-II censoring schemes are from the oldest censoring scheme. In the type-I censoring scheme, the experimenter removed the test at prefixed time; then, the failure times are random. But, in the type-II censoring scheme, the experimenter removed the test at the prefixed number of failure; then, the test time is random.

In life-testing experiments or reliability analysis, tested units fail with different modes of failure which is a common phenomenon in the life testing experiment and known by the competing risks' problem. In practice, for the life products, the product units fail under different failure modes, one of them causes failure, and the problem of assessment of the risk of any failure mode in the presence of other modes takes the attention of several authors; for more details, see [14-18] and recent [19, 20]. In our population, we record the failure time and the corresponding failure mode under consideration that only two failure modes exist and the unit fails under only one mode.

The paper aims to model type-II competing risk samples with NRMW distribution when the failure modes are independent and failure occurs under only one mode. Then, we have a description of the model mechanism and the corresponding likelihood function. Also, the model parameters are estimates for point and interval with maximum likelihood and Bayes methods. The theoretical results are discussed through simulation Monte Carlo study and real data analysis.

The paper is organized as follows. Section 2 contains some abbreviations and model description. Section 3 contains the classical estimation with the MLE method. Section 4 contains Bayes estimation with the MCMC method. In Section 5, the two lifetime data are analyzed for the illustrating purpose. Section 6 reports the results of the Monte Carlo study.

\section{The Abbreviations and Model}

In this section, we give the list of abbreviations that are used in this article as well as the complete description of the model mechanism as follows.

\subsection{List of Abbreviations}

NRMW: Reduced new modified Weibull

PDF: Probability density function

CDF: Cumulative distribution function

$S($.$) : Survival function$

HF: Hazard function

$t_{i}$ : The observed time to failure

$t_{i j}$ : $i$ th time to failure under cause $j$

$\delta_{j}$ : Indicator denoted to the cause

MCMC: Markov chain Monte Carlo

MH: Metropolis-Hastings algorithm

MSE: Mean squared error

MIL: Mean interval length

$\mathrm{CP}$ : Coverage percentage

MLE: Maximum likelihood estimate

LF: Loss function

ACI: Approximate confidence interval

CI: Credible intervals

2.2. The Model Description. Let $n$ identical independent (i.i) units are selected from population to test under the life testing experiment. And, the integer number $m$ needing for statistical inference is determined at the beginning of the experiment. When the experiment is running, failure time and the corresponding cause of failure are recorded, say $\left(T_{i}, \eta_{i}\right)_{i=1,2, \ldots, m}$. Under consideration of that only two causes 


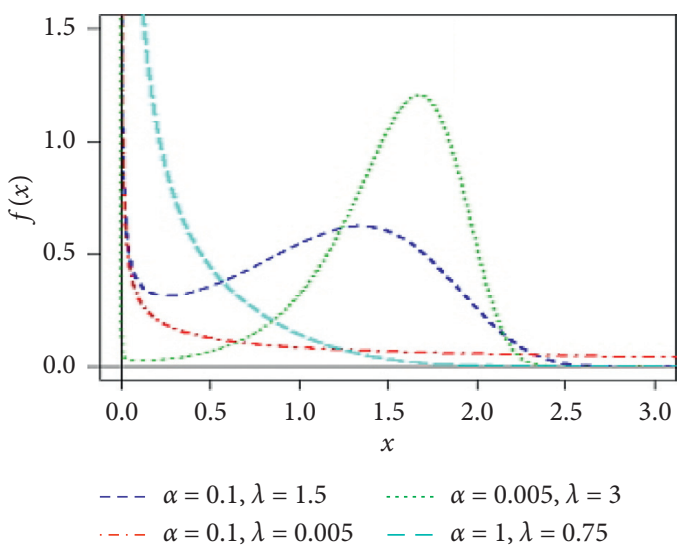

(a)

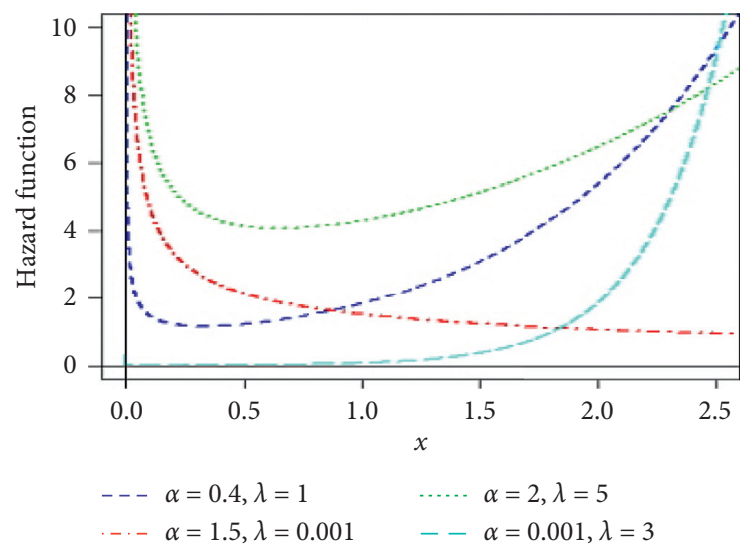

(b)

FIgURe 1: The PDF and HFR of RNMW distribution for different parameters' values.

of failure and observed sample $\mathbf{t}=\left\{\left(t_{1}, \eta_{1}\right),\left(t_{2}, \eta_{2}\right), \ldots\right.$, $\left.\left(t_{m}, \eta_{m}\right)\right\}$, the joint likelihood function is formulated by

$$
L(\underline{t} \mid \underline{\omega})=A\left(S\left(t_{m}\right)\right)_{i=1}^{n-m} \prod_{i=1}^{m}\left[h_{1}\left(t_{i}\right)\right]^{I\left(\eta_{i}=1\right)}\left[h_{2}\left(t_{i}\right)\right]^{I\left(\eta_{i}=2\right)} S\left(t_{i}\right),
$$

where $\omega=\left\{\alpha_{1}, \alpha_{2}, \lambda\right\}, A=(n ! /(n-m) !), S()=.\left(s_{1}(.) s_{2}().\right)$, and

$$
I\left(\eta_{i}=j\right)= \begin{cases}1, & \eta_{i}=j \\ 0, & \eta_{i} \neq j .\end{cases}
$$

For the given type-II competing risks' sample drawn from NRMW distributions, say $T_{i j} \sim \operatorname{NRMW}\left(\alpha_{j}, \lambda\right), j=$ 1,2 , and the observed variable $T_{i}=\min \left(T_{i 1}, T_{i 2}\right), i=1,2$, $\ldots, m$, are independent and distributed as NRMW distribution with shape parameter $\alpha_{1}+\alpha_{2}$ and accelerate parameter $\lambda$. Hence, from (8), the likelihood function is reduced to

$$
\begin{aligned}
L\left(\alpha_{1}, \alpha_{2}, \lambda \mid \underline{t}\right)= & A 2^{-m} \alpha_{1}^{m_{1}} \alpha_{2}^{m_{2}} \prod_{i=1}^{m} \frac{1}{\sqrt{t}_{i}}\left(1+\left(1+2 \lambda t_{i}\right) e^{\lambda t_{i}}\right) \\
& \times e^{-\left(\alpha_{1}+\alpha_{2}\right)}\left\{\sum_{i=1}^{m} \sqrt{t_{i}}\left\{1+e^{\lambda t_{i}}\right\}\right. \\
& \left.+(n-m) \sqrt{t_{m}}\left\{1+e^{\lambda t_{m}}\right\}\right\},
\end{aligned}
$$

where the two integers $m_{1}=\sum_{i=1}^{m} I\left(\eta_{i}=1\right)$ and $m_{2}=\sum_{i=1}^{m} I\left(\eta_{i}=2\right)$ present numbers of units that fail under first and second causes, respectively.

\section{Remarks 1}

(1) The latent failure time is distributed with NRMW distribution with $\alpha_{1}+\alpha_{2}$ and $\lambda$ shape and accelerate parameters, respectively

(2) The value $m_{1}$ is distributed with binomial distribution with sample size $m_{1}$ and probability of success $\left(\alpha_{1} / \alpha_{1}+\alpha_{2}\right)$ but $m_{1}$ is binomial distributed with sample size $m_{2}$ and probability of success $\left(\alpha_{2} / \alpha_{1}+\alpha_{2}\right)$

\section{Maximum Likelihood Estimations}

The maximum likelihood estimators of model parameters in this section are formulated for the point and interval estimate with given observed type-II competing risks' censoring sample as follows.

3.1. Point MLE. The natural logarithms of likelihood function (10) is reduced to

$$
\begin{aligned}
\ell\left(\alpha_{1}, \alpha_{2}, \lambda \mid \underline{t}\right)= & \text { Constant }+m_{1} \log \alpha_{1}+m_{2} \log \alpha_{2} \\
& +\sum_{i=1}^{m} \log \left[1+\left(1+2 \lambda t_{i}\right) e^{\lambda t_{i}}\right]-\left(\alpha_{1}+\alpha_{2}\right)\left\{\sum_{i=1}^{m} \sqrt{t_{i}}\left\{1+e^{\lambda t_{i}}\right\}+(n-m) \sqrt{t_{m}}\left\{1+e^{\lambda t_{m}}\right\}\right\} .
\end{aligned}
$$

After taking the partial derivative of the log-likelihood function with respect to parameters' vector $\alpha_{1}$ and $\alpha_{2}$, we have the likelihood equations which are defined by the following theorem. 
Theorem 1. The conditional ML estimators of parameters $\alpha_{1}$ and $\alpha_{2}$, given $\lambda>0$ and two numbers $m_{1}$ and $m_{2}>0$, can be written by

$$
\alpha_{j}(\lambda)=\frac{m_{j}}{D_{1}(\lambda)}, \quad j=1 \text { and } 2,
$$

where

$$
D_{1}(\lambda)=\sum_{i=1}^{m} \sqrt{t_{i}}\left\{1+e^{\lambda t_{i}}\right\}+(n-m) \sqrt{t_{m}}\left\{1+e^{\lambda t_{m}}\right\}
$$

Remark 2. If $m_{j}=0$, there are no failures due to cause $j$. Then, the observed data $\mathbf{t}=\left\{\left(t_{1}, \eta_{1}\right),\left(t_{2}, \eta_{2}\right), \ldots,\left(t_{m}, \eta_{m}\right)\right\}$ does not provide information about parameter $\alpha_{j}$.

Also, the partial derivative of the log-likelihood function with respect to parameter $\lambda$

$$
\sum_{i=1}^{m} \frac{2 t_{i} e^{\lambda t_{i}}+\left(1+2 \lambda t_{i}\right) t_{i} e^{\lambda t_{i}}}{1+\left(1+2 \lambda t_{i}\right) e^{\lambda t_{i}}}-\left(\alpha_{1}+\alpha_{2}\right)\left\{\sum_{i=1}^{m} t_{i} \sqrt{t_{i}} e^{\lambda t_{i}}+(n-m) t_{m} \sqrt{t_{m}} e^{\lambda t_{m}}\right\}=0 .
$$

It is clearly from (14) that there is not a closed form for $\lambda=D_{2}(\lambda)$ the ML estimator of $\lambda$; then, the iteration method can solve this problem as follows.

where

Theorem 2. The ML estimators of parameter $\lambda$ presented by the nonlinear equation can be given by

$$
D_{2}(\lambda)=\frac{\left(\left(m_{1}+m_{2}\right)\left(\sum_{i=1}^{m} t_{i} \sqrt{t_{i}} e^{\lambda t_{i}}+(n-m) t_{m} \sqrt{t_{m}} e^{\lambda t_{m}}\right) / D_{1}(\lambda)\right)-\sum_{i=1}^{m}\left(3 t_{i} e^{\lambda t_{i}} / 1+\left(1+2 \lambda t_{i}\right) e^{\lambda t_{i}}\right)}{\sum_{i=1}^{m}\left(2 t_{i}^{2} e^{\lambda t_{i}} / 1+\left(1+2 \lambda t_{i}\right) e^{\lambda t_{i}}\right)} .
$$

Remark 3. Nonlinear equation (16) can be solved with any iteration method such as Newton-Raphson or fixed point but needs to find the initial value of $\lambda$ which can be obtained from the profile likelihood function presented by

$$
g(\lambda \mid \underline{t}) \propto \sum_{j=1}^{2} m_{j} \log \left[\frac{m_{j}}{D_{1}(\lambda)}\right]+\sum_{i=1}^{m} \log \left[1+\left(1+2 \lambda t_{i}\right) e^{\lambda t_{i}}\right]-\left(m_{1}+m_{2}\right)
$$

Then, from theorems (1) and (2), the ML estimate of the parameters' vector $\omega=\left(\alpha_{1}, \alpha_{2}, \lambda\right)$ is obtained to be $\widehat{\omega}=\left(\widehat{\alpha}_{1}, \widehat{\alpha}_{2}, \widehat{\lambda}\right)$.
3.2. Interval Estimation. The second derivative of the $\log$ likelihood function with respect to $\omega=\left(\alpha_{1}, \alpha_{2}, \lambda\right)$ is given by

$$
\begin{aligned}
\frac{\partial^{2} \ell\left(\alpha_{1}, \alpha_{2}, \lambda \mid \underline{t}\right)}{\partial \alpha_{j}^{2}}= & \frac{-m_{j}}{\alpha_{j}}, \quad i=1 \text { and } 2, \\
\frac{\partial^{2} \ell\left(\alpha_{1}, \alpha_{2}, \lambda \mid \underline{t}\right)}{\partial \lambda^{2}}= & \sum_{i=1}^{m} \frac{D_{3}(\lambda)(d / \mathrm{d} \lambda) D_{3}(\lambda)-\left[(d / \mathrm{d} \lambda) D_{3}(\lambda)\right]^{2}}{D_{3}^{2}(\lambda)} \\
& -\left(\alpha_{1}+\alpha_{2}\right)\left\{\sum_{i=1}^{m} t_{i}^{2} \sqrt{t_{i}} e^{\lambda t_{i}}+(n-m) t_{m}^{2} \sqrt{t_{m}} e^{\lambda t_{m}}\right\}, \\
\frac{\partial^{2} \ell\left(\alpha_{1}, \alpha_{2}, \lambda \mid \underline{t}\right)}{\partial \alpha_{1} \partial \alpha_{2}}= & \frac{\partial^{2} \ell\left(\alpha_{1}, \alpha_{2}, \lambda \mid \underline{t}\right)}{\partial \alpha_{2} \partial \alpha_{1}}=0,
\end{aligned}
$$




$$
\begin{aligned}
\frac{\partial^{2} \ell\left(\alpha_{1}, \alpha_{2}, \lambda \mid \underline{t}\right)}{\partial \alpha_{1} \partial \lambda} & =\frac{\partial^{2} \ell\left(\alpha_{1}, \alpha_{2}, \lambda \mid \underline{t}\right)}{\partial \lambda \partial \alpha_{1}}=\frac{\partial^{2} \ell\left(\alpha_{1}, \alpha_{2}, \lambda \mid \underline{t}\right)}{\partial \alpha_{2} \partial \lambda}=\frac{\partial^{2} \ell\left(\alpha_{1}, \alpha_{2}, \lambda \mid \underline{t}\right)}{\partial \lambda \partial \alpha_{2}} \\
& =-\left\{\sum_{i=1}^{m} t_{i} \sqrt{t_{i}} e^{\lambda t_{i}}+(n-m) t_{m} \sqrt{t_{m}} e^{\lambda t_{m}}\right\},
\end{aligned}
$$

where

$$
D_{3}(\lambda)=1+\left(1+2 \lambda t_{i}\right) e^{\lambda t_{i}}
$$

Hence, from equations (19) to (21), the Fisher information matrix (IM) is defined as minus expectation of equations (19)-(21) to be given by

$$
\mathrm{IM}=\mathrm{E}\left(\begin{array}{c}
-\frac{\partial^{2} \ell\left(\alpha_{1}, \alpha_{2}, \lambda \mid \underline{t}\right)}{\partial \alpha_{1}^{2}}-\frac{\partial^{2} \ell\left(\alpha_{1}, \alpha_{2}, \lambda \mid \underline{t}\right)}{\partial \alpha_{1} \partial \alpha_{2}}-\frac{\partial^{2} \ell\left(\alpha_{1}, \alpha_{2}, \lambda \mid \underline{t}\right)}{\partial \alpha_{1} \partial \lambda} \\
-\frac{\partial^{2} \ell\left(\alpha_{1}, \alpha_{2}, \lambda \mid \underline{t}\right)}{\partial \alpha_{2} \partial \alpha_{1}}-\frac{\partial^{2} \ell\left(\alpha_{1}, \alpha_{2}, \lambda \mid \underline{t}\right)}{\partial \alpha_{2}^{2}}-\frac{\partial^{2} \ell\left(\alpha_{1}, \alpha_{2}, \lambda \mid \underline{t}\right)}{\partial \alpha_{2} \partial \lambda} \\
-\frac{\partial^{2} \ell\left(\alpha_{1}, \alpha_{2}, \lambda \mid \underline{t}\right)}{\partial \lambda \partial \alpha_{1}}-\frac{\partial^{2} \ell\left(\alpha_{1}, \alpha_{2}, \lambda \mid \underline{t}\right)}{\partial \lambda \partial \alpha_{2}}-\frac{\partial^{2} \ell\left(\alpha_{1}, \alpha_{2}, \lambda \mid \underline{t}\right)}{\partial \lambda^{2}}
\end{array}\right) .
$$

The approximate value of the Fisher information matrix is defined by

$$
\mathrm{AIM}=\mathrm{IM}_{0}=\left(\begin{array}{c}
-\frac{\partial^{2} \ell\left(\alpha_{1}, \alpha_{2}, \lambda \mid \underline{t}\right)}{\partial \alpha_{1}^{2}}-\frac{\partial^{2} \ell\left(\alpha_{1}, \alpha_{2}, \lambda \mid \underline{t}\right)}{\partial \alpha_{1} \partial \alpha_{2}}-\frac{\partial^{2} \ell\left(\alpha_{1}, \alpha_{2}, \lambda \mid \underline{t}\right)}{\partial \alpha_{1} \partial \lambda} \\
-\frac{\partial^{2} \ell\left(\alpha_{1}, \alpha_{2}, \lambda \mid \underline{t}\right)}{\partial \alpha_{2} \partial \alpha_{1}}-\frac{\partial^{2} \ell\left(\alpha_{1}, \alpha_{2}, \lambda \mid \underline{t}\right)}{\partial \alpha_{2}^{2}}-\frac{\partial^{2} \ell\left(\alpha_{1}, \alpha_{2}, \lambda \mid \underline{t}\right)}{\partial \alpha_{2} \partial \lambda} \\
-\frac{\partial^{2} \ell\left(\alpha_{1}, \alpha_{2}, \lambda \mid \underline{t}\right)}{\partial \lambda \partial \alpha_{1}}-\frac{\partial^{2} \ell\left(\alpha_{1}, \alpha_{2}, \lambda \mid \underline{t}\right)}{\partial \lambda \partial \alpha_{2}}-\frac{\partial^{2} \ell\left(\alpha_{1}, \alpha_{2}, \lambda \mid \underline{t}\right)}{\partial \lambda^{2}}
\end{array}\right)_{a t\left(\widehat{\alpha}_{1}, \widehat{\alpha}_{2}, \widehat{\lambda}\right)} .
$$

Under limiting properties of the MLEs is using it to present the asymptotic distribution of $\widehat{\omega}=\left(\widehat{\alpha}_{1}, \widehat{\alpha}_{2}, \widehat{\lambda}\right)$. Then, the asymptotically normally distributed mean $\omega=\left(\alpha_{1}, \alpha_{2}, \lambda\right)$ and variance covariance matrix $\operatorname{IM}_{0(\hat{\omega})}^{-1}$ describe the asymptotic distribution of $\omega=\left(\alpha_{1}, \alpha_{2}, \lambda\right)$ Therefore, the interval estimate with confidence level $100(1-2 \gamma) \%$ is given by

$$
\widehat{\alpha}_{1} \mp Z_{\gamma} \sqrt{\epsilon_{11}}, \widehat{\alpha}_{2} \mp Z_{\gamma} \sqrt{\epsilon_{22}} \text {, and } \widehat{\lambda} \mp Z_{\gamma} \sqrt{\epsilon_{33}} \text {, }
$$

where the vector $\left(\epsilon_{11}, \epsilon_{22}, \epsilon_{33}\right)$ presents the diagonal of inverse of $\mathrm{IM}_{0(\underline{\hat{\omega}})^{-1}}$ and the value $Z_{\gamma}$ presents the percentile of the standard normal distribution with right-tail probability $\gamma$.

\section{MCMC Bayes Estimation}

Bayesian estimation depends on the amount of the past information about the model parameters which is known by prior distribution. And, the amount of the information existing in data is known by the likelihood function. Then, we aim to formulate these two data in the form of probability distribution, known by posterior distribution, as follows.

Suppose that the parameters' vector $\omega=\left(\alpha_{1}, \alpha_{2}, \lambda\right)$ is distributed with independent gamma priors. Therefore, the joint prior distribution can be presented by 


$$
\pi^{*}\left(\alpha_{1}, \alpha_{2}, \lambda\right) \propto \alpha_{1}^{a_{1}-1} \alpha_{2}^{a_{2}-1} \lambda^{a_{3}-1} \exp \left(-b_{1} \alpha_{1}-b_{2} \alpha_{2}-b_{3} \lambda\right), \quad a_{i} \text { and } b_{i}>0 \text { and } i=1,2 \text {, and } 3 \text {. }
$$
by

Generally, the posterior distribution can be formulated

$$
\pi\left(\left(\alpha_{1}, \alpha_{2}, \lambda \mid \underline{t}\right)\right)=\frac{\pi^{*}\left(\alpha_{1}, \alpha_{2}, \lambda\right) L\left(\alpha_{1}, \alpha_{2}, \lambda \mid \underline{t}\right)}{\iint_{\underline{\omega}} \int \pi^{*}\left(\alpha_{1}, \alpha_{2}, \lambda\right) L\left(\alpha_{1}, \alpha_{2}, \lambda \mid \underline{t}\right) \mathrm{d} \alpha_{1} \mathrm{~d} \alpha_{2} \mathrm{~d} \lambda}
$$

Also, generally the ratio of two integrals (26), especially,

$$
\widehat{\Omega}_{B}=\iint_{\underline{\omega}} \int \Omega(\underline{\omega}) \pi(\underline{\omega} \mid \underline{t}) \mathrm{d} \alpha_{1} \mathrm{~d} \alpha_{2} \mathrm{~d} \lambda
$$
in the high-dimensional case, cannot obtain analytical. Then, different methods are available to approximate this ratio such as Lindley approximation and numerical integration. But, one of the most applied methods known by MCMC method is discussed in this article. Hence, without loss of the generality, the Bayes estimate of the function $\Omega(\underline{\omega})$ under squared error loss (SEL) function is given by

$$
\begin{aligned}
\pi(\underline{\boldsymbol{\omega}} \mid \underline{t})= & C \alpha_{1}^{m_{1}+a_{1}-1} \alpha_{2}^{m_{2}+a_{2}-1} \lambda^{a_{3}-1} \alpha_{1}^{m_{1}} \alpha_{2}^{m_{2}} \prod_{i=1}^{m}\left(1+\left(1+2 \lambda t_{i}\right) e^{\lambda t_{i}}\right) \\
& \times e^{\left\{-\left(\alpha_{1}+\alpha_{2}\right)\left(\sum_{i=1}^{m} \sqrt{t_{i}}\left\{1+e^{\lambda t_{i}}\right\}+(n-m) \sqrt{t_{m}}\left\{1+e^{\lambda t_{m}}\right\}\right)-b_{1} \alpha_{1}-b_{2} \alpha_{2}-b_{3} \lambda\right\}},
\end{aligned}
$$

where $C$ is the normalized constant. From the posterior distribution the full-conditional PDFs of the vector $\omega, \omega=$ $\left(\alpha_{1}, \alpha_{2}, \lambda\right)$ is given by

$$
\begin{aligned}
& \pi_{1}\left(\alpha_{1} \mid \alpha_{2}, \lambda, \underline{t}\right) \propto \operatorname{Gamma}\left(m_{1}+a_{1}, b_{1}+\sum_{i=1}^{m} \sqrt{t_{i}}\left\{1+e^{\lambda t_{i}}\right\}+(n-m) \sqrt{t_{m}}\left\{1+e^{\lambda t_{m}}\right\}\right), \\
& \pi_{2}\left(\alpha_{2} \mid \alpha_{1}, \lambda, \underline{t}\right) \propto \operatorname{Gamma}\left(m_{2}+a_{2}, b_{2}+\sum_{i=1}^{m} \sqrt{t_{i}}\left\{1+e^{\lambda t_{i}}\right\}+(n-m) \sqrt{t_{m}}\left\{1+e^{\lambda t_{m}}\right\}\right), \\
& \pi_{3}\left(\lambda \mid \alpha_{1}, \alpha_{2}, \underline{t}\right) \propto \lambda^{a_{3}-1} e^{\left\{\sum_{i=1}^{m}\left(\log \left[\left(1 / \sqrt{t_{i}}\right)\left(1+\left(1+2 \lambda t_{i}\right) e^{\lambda t_{i}}\right)\right]-\left(\alpha_{1}+\alpha_{2}\right)\left(\sqrt{t_{i}}\left\{1+e^{\lambda t_{i}}\right\}+(n-m) \sqrt{t_{m}}\left\{1+e^{\lambda t_{m}}\right\}\right)\right)-b_{3} \lambda\right\} .} .
\end{aligned}
$$

The full-conditional PDFs have shown that generation from posterior distribution depend on two-conditional gamma density and more with general conditional function which is similar to normal distribution. Hence, the $\mathrm{MH}$ algorithm under Gibbs is a more suitable algorithm to construct the empirical posterior distribution (see [24]) 
Also, for the recent review of $\mathrm{MH}$ algorithms under Gibbs, see [25] and [26].

\subsection{Gibbs with $\mathrm{MH}$ Algorithms}

Step 1: set the indicator $\kappa=1$ and $\omega^{(0)}=\left(\widehat{\alpha}_{1}, \widehat{\alpha}_{2}, \widehat{\lambda}\right)$. Step 2: from (28) and (29), generate $\alpha_{1}^{(\kappa)}$ and $\alpha_{2}^{(\kappa)}$.

Step 3: with normal proposal distribution with mean $\lambda^{(\kappa-1)}$ and variance $V(\lambda)=\epsilon_{33}$, generate the value $\lambda^{(\kappa)}$ under proposal normal distribution with $\mathrm{MH}$ algorithms as follows.

(i) Generate the proposal, $\lambda^{*}$, from $N\left(\lambda^{(\kappa-1)}, \epsilon_{33}\right)$.

(ii) Calculate the acceptance probability:

$$
\sigma\left(\lambda^{(\kappa-1)}, \lambda^{*}\right)=\min \left[1, \frac{\pi_{3}\left(\lambda^{*}\right)}{\lambda^{(\kappa-1)}}\right] .
$$

(iii) Generate uniform $(0,1)$ value $U$.

(iv) If $U \leq \sigma\left(\lambda^{(\kappa-1)}, \lambda^{*}\right)$, accept the proposal $\lambda^{*}$. Otherwise, reject the proposal and repeat the value $\lambda^{(\kappa-1)}$.

Step 4: report $\omega^{(\kappa)}=\left(\alpha_{1}^{(\kappa)}, \alpha_{2}^{(\kappa)}, \lambda^{(\kappa)}\right)$ and set $\kappa=\kappa+1$. Step 5: steps from (2) to (4) are repeated $N$ times.

Step 6: the point Bayes estimators for any function $\Omega(\underline{\omega})$ are defined by

$$
\widehat{\Omega}_{B}=\frac{1}{N-M}_{i=M+1}^{N} \Omega^{(i)},
$$

and integer $M$ is the number needed to achieve the stationary distribution (burn-in). The corresponding posterior variance of $\Omega(\underline{\omega})$ is given by

$$
\widehat{V}(\Omega)=\frac{1}{N-M}_{i=M+1}^{N}\left(\Omega^{(i)}-\widehat{\Omega}_{B}\right)^{2} .
$$

Step 7: for interval estimators with confidence level $100(1-2 \gamma) \%$ of $\Omega(\underline{\omega})$ is given by

$$
\left(\Omega_{\gamma(N-M)}, \Omega_{(1-\gamma)(N-M)}\right)
$$

\section{Data Analysis}

In this section, we discussed two lifetime datasets; real and simulated data to illustrate the developed results in this paper.

5.1. Example 1: Real Dataset. Under a laboratory experiment with a conventional laboratory environment, Hoel [23] tested a male mice exposed to radiation dose of 300 roentgens at age of 5-6 weeks. The lifetime data under two causes of failure, thymic lymphoma as a first cause and other causes as a second cause of failure, are reported in Table 1. The causes of failure are obtained for each mouse by autopsy. Restricting the analysis to two causes of death, for the purpose of analysis, we consider thymic lymphoma as cause
1 and reticulum cell sarcoma as cause 2. Different combinations of Hoel data are used by several authors [24-26]. Hence, under sample size $n=61$ and effected sample size $m=23$, the type-II competing risks' data after divided by 100 for simplicity are given by $\mathrm{t}=\{(0.40,2),(0.42,2),(0.51,2)$, $(0.62,2),(1.59,1),(1.63,2),(1.79,2),(1.89,1),(1.91,1),(1.98$. $1),(2.00,1),(2.06,2),(2.07,1),(2.20,1),(2.22,2),(2.28,2)$, $(2.35,1),(2.45,1),(2.49,2),(2.50,1),(2.52,2),(2.56,1)$, $(2.61,1)\}$.

For given $t$ dataset, the efficiency of the theoretical results of NRMW distribution for analysis of this data is constructed. Under type-II competing risks' data, the profile loglikelihood function of $\lambda$ given in Figure 2 is a unimodal function, and the ML estimate is computed with iteration with initial guess of $\lambda$ as 1.0. The noninformative prior is used for prior information as $a_{i}=b_{i}=0.0001$, $i=1,2,3$, and 4 . The results of MLE and Bayes estimate are reported in Table 2 for point and 95\% interval estimate. For the MCMC approach in the Bayes method. We run the chan (Gibbs with MH algorithm) 11000 times and the first 1000 times which are needed to reach the stationary distribution are deleted as known by "brun-in." The empirical posterior distribution under the MCMC approach is described with Figures 3 and 4 . The MCMC convergence to the posterior distribution is described by Figures 3 and 4 .

5.2. Example 2: Simulated Dataset. The algorithms used to generate and analyze the type-II competing risks sample from NRMW distribution is described as follows:

(1) Suppose a sample size $n=50$ and effected sample size $m=30$.

(2) From gamma prior distributions with $\left.\left(a_{i}, b_{i}\right)\right|_{i=1,2,3}=\{(1,5),(1,15),(5,2)\}, \quad$ generate a sample of size 10 . Then, the true parameters' value is computed to be the sample mean $\omega=\left\{\alpha_{1}, \alpha_{2}, \lambda\right\}=\{0.2,0.05,2.2\}$.

(3) Generate type-II competing risks' sample from NRMW with $\left(\alpha_{1}+\alpha_{2}\right)$ shape and $\lambda$ scale parameters to be $\mathbf{t}=\{0.0273,0.0349,0.0604,0.0779,0.0834$, $0.0902,0.1604,0.167,0.1674,0.1682,0.1892,0.2384$, $0.2431,0.2786,0.2786,0.2958,0.3827,0.452,0.5049$, $0.5735,0.5845,0.5996,0.6032,0.6201,0.6279,0.6376$, $0.6461,0.6529,0.6668,0.6726\} ; m_{1}$ and $m_{2}$ are generated from binomial distribution to be $m_{1}=25$ and $m_{2}=5$.

(4) The graphs of profile log-likelihood function and empirical posterior distribution under MCMC are given in Figures 5-7, respectively.

(5) The results of ML and Bayes estimate are reported in Table 3 for point and 95\% interval estimate.

\section{Simulation Studies}

The quality of the proposed model and the corresponding developed results in this paper is assessed through the Monte Carlo simulation study as follows. In our study, we measure the effect of change of each sample size $n$ affect sample size $m$ 
TABLe 1: The failure data of 61 male mice under radiation dose or $300 \mathrm{r}$ at age 5-6 weeks.

\begin{tabular}{lccccccccccc}
\hline Thymic & 159 & 189 & 191 & 198 & 200 & 207 & 220 & 235 & 245 & 250 & 256 \\
lymphoma & 261 & 265 & 266 & 280 & 343 & 356 & 383 & 403 & 414 & 428 & 432 \\
& 40 & 42 & 51 & 62 & 163 & 179 & 206 & 222 & 228 & 252 & 249 \\
Other causes & 282 & 324 & 333 & 341 & 366 & 385 & 407 & 420 & 431 & 441 & 461 \\
& 462 & 482 & 517 & 517 & 524 & 564 & 567 & 586 & 619 & 620 & 621 \\
& 622 & 647 & 651 & 686 & 761 & 763 & & \\
\hline
\end{tabular}

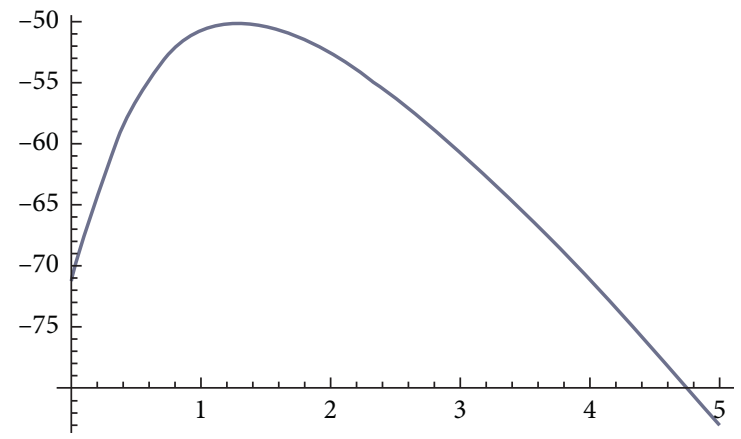

Figure 2: Profile log-likelihood function of $\lambda$.

TABLe 2: Point and 95\% interval ML and Bayes estimate.

\begin{tabular}{lcccccc}
\hline Pa. & $(\cdot)_{\mathrm{ML}}$ & $(\cdot)_{\mathrm{B}-\mathrm{MCMC}}$ & $95 \%$ ACI & Length & $95 \%$ CI & Length \\
\hline$\alpha_{1}$ & 0.00512 & 0.0068 & $(0.00000,0.01472)$ & 0.0147 & $(0.0012,0.0183)$ & 0.0172 \\
$\alpha_{2}$ & 0.00469 & 0.0062 & $(0.00000,0.01512)$ & 0.0151 & $(0.0011,0.0171)$ & 0.0161 \\
$\lambda$ & 1.28389 & 1.2411 & $(0.54999,2.01780)$ & 1.4678 & $(0.7868,1.8240)$ & 1.0372 \\
\hline
\end{tabular}

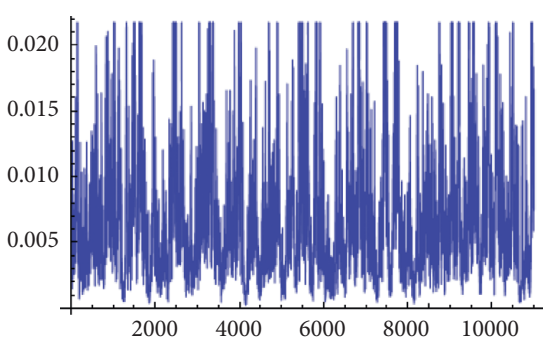

(a)

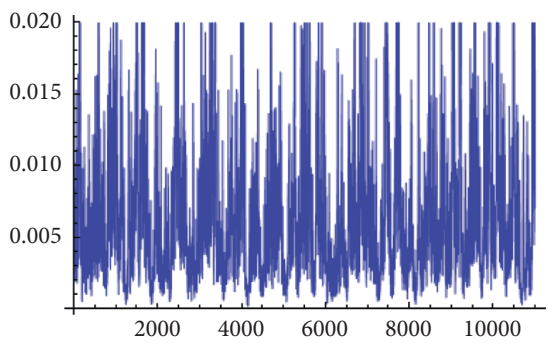

(b)

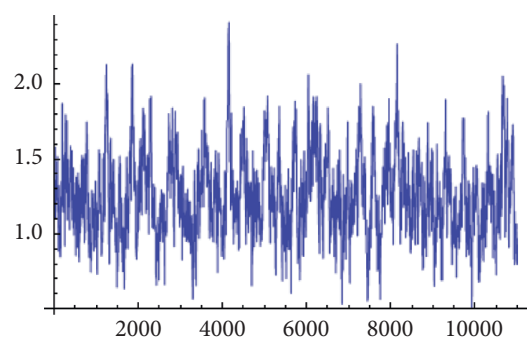

(c)

Figure 3: Simulation number generated by the MCMC method for $\alpha_{1}, \alpha_{2}$, and $\lambda$, respectively.

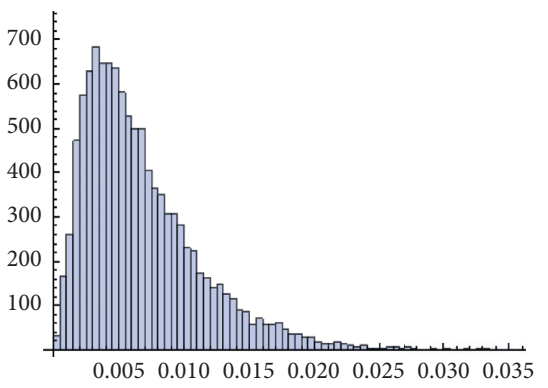

(a)

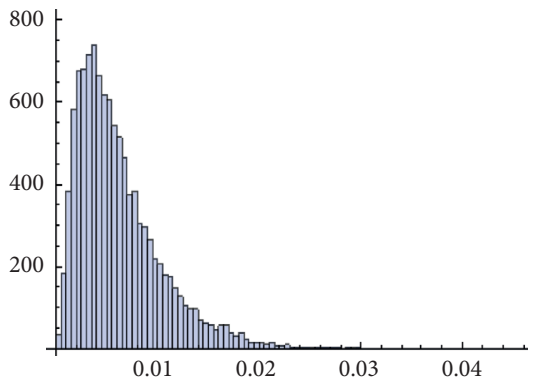

(b)

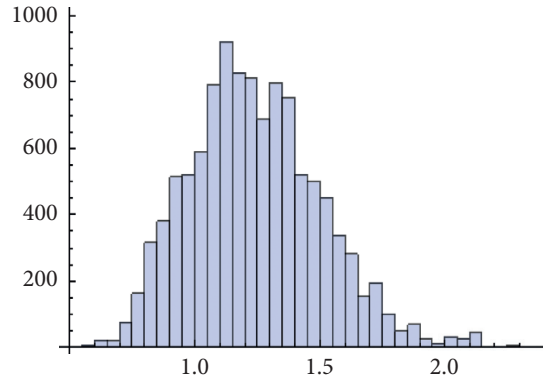

(c)

FIgURE 4: Histogram generated by the MCMC method for $\alpha_{1}, \alpha_{2}$, and $\lambda$, respectively. 


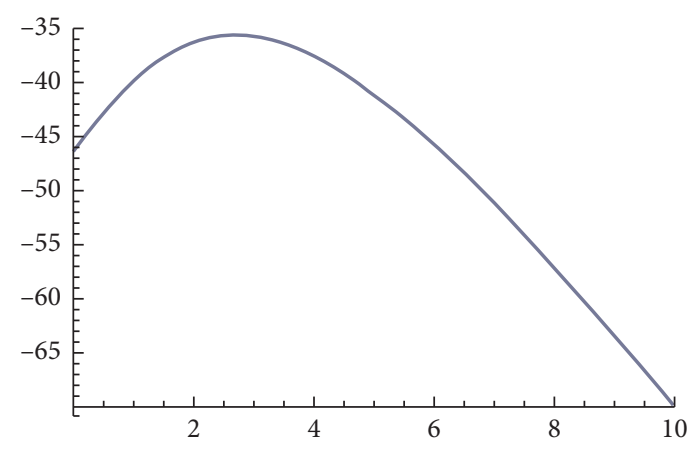

Figure 5: Profile log-likelihood function of $\lambda$.

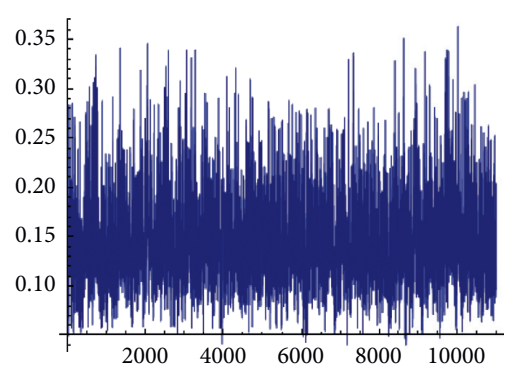

(a)

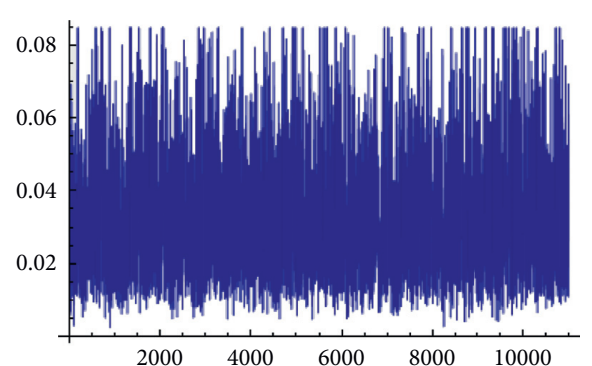

(b)

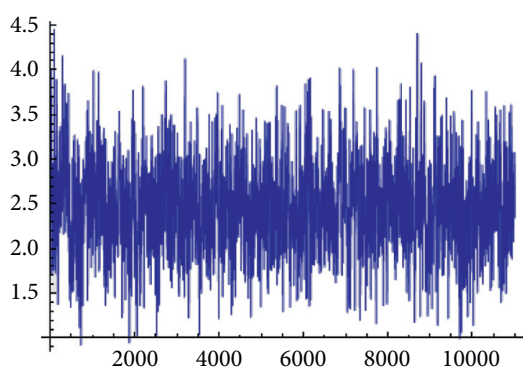

(c)

FIgURE 6: Simulation number generated by the MCMC method for $\alpha_{1}, \alpha_{2}$, and $\lambda$, respectively.

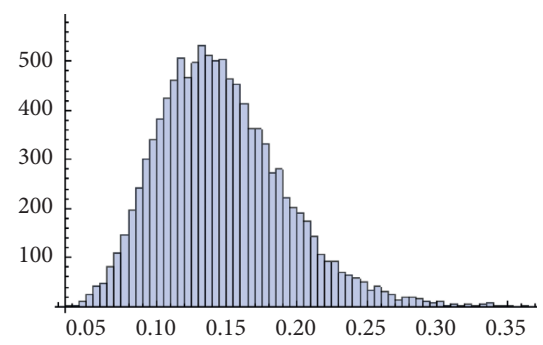

(a)

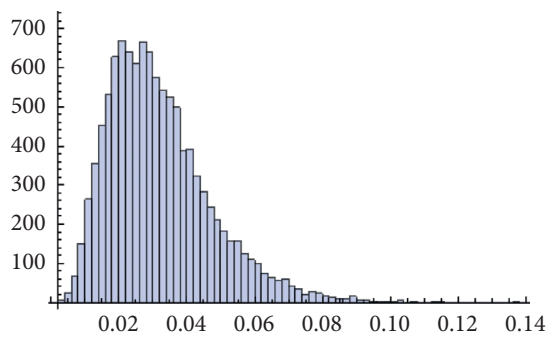

(b)

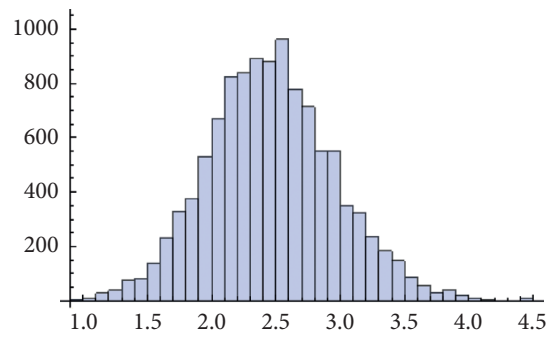

(c)

Figure 7: Histogram generated by the MCMC method for $\alpha_{1}, \alpha_{2}$, and $\lambda$, respectively.

and model parameters $\omega=\left\{\alpha_{1}, \alpha_{2}, \lambda\right\}$. So, different combinations of the censoring scheme are reported in the simulation table. For the parameters' values, two sets are used $\omega=\{\{0.01,0.02,1.5\},\{1.0,1.5,2.0\}\}$. The simulation study is carried out with respect to 1000 simulated datasets. The prior parameters are selected to satisfy the property that $E\left(\omega_{i}\right) \simeq\left(a_{i} / b_{i}\right)$. For the point estimate, we compute mean (ME) and the square root of mean squared error (MSE). For the interval estimate, we compute mean interval length (MIL) and probability coverage (PC). We run chan (MCMC iteration) 11000 times discarding the first 1000 values as brun-in (number of iterations needed to reach the stationary distribution). The results of the simulation study are reported in Tables 4-7.

Models with the bathtub-shaped or increasing failure rate function have modeled a different lifetime data. In this section, we adopted the simulation study for one reducing form of the modified Weibull distribution. 
TABle 3: Point and 95\% interval ML and Bayes estimate.

\begin{tabular}{|c|c|c|c|c|c|c|}
\hline $\mathrm{Pa}$. & $(\cdot)_{\mathrm{ML}}$ & $(\cdot)_{\mathrm{B}-\mathrm{MCMC}}$ & $95 \%$ ACI & Length & $95 \%$ CI & Length \\
\hline$\alpha_{1}=0.20$ & 0.1274 & 0.1467 & $(0.0334,0.2215)$ & 0.1881 & $(0.0742,0.2469)$ & 0.1727 \\
\hline$\alpha_{2}=0.05$ & 0.0255 & 0.0320 & $(0.0000,0.0529)$ & 0.0529 & $(0.0104,0.0690)$ & 0.0586 \\
\hline$\lambda=2.20$ & 1.2839 & 2.4580 & $(1.4654,3.8878)$ & 2.4224 & $(1.5523,3.4745)$ & 1.9222 \\
\hline
\end{tabular}

TABle 4: MEs and MSEs from $\omega=\{0.01,0.02,1.5\}$.

\begin{tabular}{|c|c|c|c|c|c|c|c|c|c|c|}
\hline \multirow{2}{*}{$(n, m)$} & \multirow{2}{*}{ Tolls } & \multicolumn{3}{|c|}{ MLE } & \multicolumn{3}{|c|}{ Bayes $\left(P_{0}\right)$} & \multicolumn{3}{|c|}{ Bayes $\left(P_{1}\right)$} \\
\hline & & $\alpha$ & $\beta$ & $\lambda$ & $\alpha$ & $\beta$ & $\lambda$ & $\alpha$ & $\beta$ & $\lambda$ \\
\hline \multirow{2}{*}{$(30,10)$} & MEs & 0.0135 & 0.0254 & 1.8420 & 0.0128 & 0.0245 & 1.8151 & 0.0119 & 0.0225 & 1.6241 \\
\hline & MSEs & 0.0075 & 0.0125 & 0.3750 & 0.0073 & 0.0122 & 0.3401 & 0.0054 & 0.0109 & 0.2841 \\
\hline \multirow{2}{*}{$(30,20)$} & MEs & 0.0120 & 0.0245 & 1.8180 & 0.0117 & 0.0241 & 1.8070 & 0.0115 & 0.0214 & 1.6110 \\
\hline & MSEs & 0.0060 & 0.0113 & 0.3501 & 0.0059 & 0.0109 & 0.3182 & 0.0040 & 0.0095 & 0.2480 \\
\hline \multirow{2}{*}{$(30,30)$} & MEs & 0.0108 & 0.0235 & 1.7010 & 0.0109 & 0.0237 & 1.7021 & 0.0111 & 0.0212 & 1.6030 \\
\hline & MSEs & 0.0045 & 0.0101 & 0.3322 & 0.0042 & 0.0100 & 0.3250 & 0.0032 & 0.0068 & 0.2171 \\
\hline \multirow{2}{*}{$(50,15)$} & MEs & 0.0123 & 0.0247 & 1.8241 & 0.0118 & 0.0239 & 1.8110 & 0.0113 & 0.0212 & 1.6132 \\
\hline & MSEs & 0.0062 & 0.0115 & 0.3541 & 0.0058 & 0.0111 & 0.3210 & 0.0041 & 0.0092 & 0.2491 \\
\hline \multirow{2}{*}{$(50,30)$} & MEs & 0.0109 & 0.0234 & 1.7040 & 0.0111 & 0.0235 & 1.7030 & 0.0109 & 0.0214 & 1.6051 \\
\hline & MSEs & 0.0041 & 0.0099 & 0.3291 & 0.0039 & 0.0980 & 0.326 & 0.0028 & 0.0059 & 0.2131 \\
\hline \multirow{2}{*}{$(50,50)$} & MEs & 0.0098 & 0.0211 & 1.6871 & 0.0101 & 0.0217 & 1.6551 & 0.0097 & 0.0221 & 1.5550 \\
\hline & MSEs & 0.0028 & 0.0070 & 0.3021 & 0.0025 & 0.0680 & 0.2890 & 0.0015 & 0.0048 & 0.1900 \\
\hline \multirow{2}{*}{$(70,35)$} & MEs & 0.0120 & 0.0218 & 1.6980 & 0.0113 & 0.0225 & 1.6602 & 0.0102 & 0.0228 & 1.5672 \\
\hline & MSEs & 0.0037 & 0.0082 & 0.3130 & 0.0034 & 0.0751 & 0.2941 & 0.0024 & 0.0051 & 0.1971 \\
\hline \multirow{2}{*}{$(70,50)$} & MEs & 0.0101 & 0.0212 & 1.689 & 0.0102 & 0.0218 & 1.6510 & 0.0096 & 0.0219 & 1.552 \\
\hline & MSEs & 0.0024 & 0.0068 & 0.3040 & 0.0023 & 0.0641 & 0.2870 & 0.0013 & 0.0042 & 0.1910 \\
\hline \multirow{2}{*}{$(70,70)$} & MEs & 0.0099 & 0.0208 & 1.6031 & 0.0099 & 0.0210 & 1.555 & 0.0098 & 0.0219 & 1.5400 \\
\hline & MSEs & 0.0014 & 0.0059 & 0.2750 & 0.0011 & 0.0551 & 0.2710 & 0.0009 & 0.0035 & 0.1641 \\
\hline
\end{tabular}

TABle 5: MILs and CPs from $\omega=\{0.01,0.02,1.5\}$.

\begin{tabular}{lcccccccccc}
\hline \multirow{2}{*}{$(n, m)$} & Tolls & & MLE & & \multicolumn{3}{c}{ Bayes $\left(P_{0}\right)$} & & \multicolumn{3}{c}{ Bayes $\left(P_{1}\right)$} \\
& & $\alpha$ & $\beta$ & $\lambda$ & $\alpha$ & $\beta$ & $\lambda$ & $\alpha$ & $\beta$ & 0.0422 \\
$(30,10)$ & MILs & 0.0299 & 0.0475 & 3.4152 & 0.0287 & 0.0464 & 3.4149 & 0.0215 & 3.4109 \\
& CPs & $(0.89)$ & $(0.88)$ & $(0.89)$ & $(0.90)$ & $(0.89)$ & $(0.90)$ & $(0.90)$ & $(0.89)$ & $(0.90)$ \\
\hline \multirow{2}{*}{$(30,20)$} & MILs & 0.0268 & 0.0451 & 3.4127 & 0.0254 & 0.0431 & 3.4122 & 0.0185 & 0.0401 & 3.4090 \\
& CPs & $(0.90)$ & $(0.90)$ & $(0.92)$ & $(0.91)$ & $(0.96)$ & $(0.92)$ & $(0.93)$ & $(0.91)$ & $(0.92)$ \\
\hline \multirow{2}{*}{$(30,30)$} & MILs & 0.0237 & 0.0419 & 3.4100 & 0.0221 & 0.0402 & 3.4100 & 0.0157 & 0.0370 & 3.4065 \\
& CPs & $(0.93)$ & $(0.91)$ & $(0.94)$ & $(0.93)$ & $(0.95)$ & $(0.93)$ & $(0.92)$ & $(0.95)$ & $(0.94)$ \\
\hline \multirow{2}{*}{$50,15)$} & MILs & 0.0272 & 0.0457 & 3.4131 & 0.0255 & 0.0434 & 3.4127 & 0.0190 & 0.0408 & 3.4092 \\
& CPs & $(0.90)$ & $(0.89)$ & $(0.90)$ & $(0.91)$ & $(0.90)$ & $(0.91)$ & $(0.91)$ & $(0.91)$ & $(0.90)$ \\
\hline \multirow{2}{*}{$50,30)$} & MILs & 0.0241 & 0.0415 & 3.4101 & 0.0219 & 0.0408 & 3.4103 & 0.0155 & 0.0372 & 3.4061 \\
& CPs & $(0.92)$ & $(0.92)$ & $(0.93)$ & $(0.91)$ & $(0.91)$ & $(0.93)$ & $(0.90)$ & $(0.94)$ & $(0.93)$ \\
\hline \multirow{2}{*}{$(50,50)$} & MILs & 0.0225 & 0.0403 & 3.4085 & 0.0207 & 0.0400 & 3.4088 & 0.0142 & 0.0361 & 3.4048 \\
& CPs & $(0.93)$ & $(0.90)$ & $(0.92)$ & $(0.93)$ & $(0.93)$ & $(0.95)$ & $(0.93)$ & $(0.92)$ & $(0.96)$ \\
\hline \multirow{2}{*}{$(70,35)$} & MILs & 0.0231 & 0.0409 & 3.4092 & 0.0214 & 0.0407 & 3.4097 & 0.0149 & 0.0372 & 3.4054 \\
& CPs & $(0.90)$ & $(0.91)$ & $(0.92)$ & $(0.92)$ & $(0.93)$ & $(0.90)$ & $(0.93)$ & $(0.94)$ & $(0.92)$ \\
\hline \multirow{2}{*}{$(70,50)$} & MILs & 0.0225 & 0.0401 & 3.4082 & 0.0204 & 0.0402 & 3.4083 & 0.0144 & 0.0365 & 3.4045 \\
& CPs & $(0.92)$ & $(0.92)$ & $(0.93)$ & $(0.94)$ & $(0.93)$ & $(0.94)$ & $(0.97)$ & $(0.93)$ & $(0.95)$ \\
\hline \multirow{2}{*}{$(70,70)$} & MILs & 0.0209 & 0.0382 & 3.4041 & 0.0179 & 0.0376 & 3.4045 & 0.0112 & 0.0331 & 3.4015 \\
& CPs & $(0.94)$ & $(0.90)$ & $(0.95)$ & $(0.93)$ & $(0.93)$ & $(0.92)$ & $(0.93)$ & $(0.92)$ & $(0.95)$ \\
\hline
\end{tabular}


TABle 6: MEs and MSEs from $\omega=\{0.1,0.05,1.0\}$.

\begin{tabular}{|c|c|c|c|c|c|c|c|c|c|c|}
\hline \multirow{2}{*}{$(n, m)$} & \multirow{2}{*}{ Tolls } & \multicolumn{3}{|c|}{ MLE } & \multicolumn{3}{|c|}{ Bayes $\left(P_{0}\right)$} & \multicolumn{3}{|c|}{ Bayes $\left(P_{1}\right)$} \\
\hline & & $\alpha$ & $\beta$ & $\lambda$ & $\alpha$ & $\beta$ & $\lambda$ & $\alpha$ & $\beta$ & $\lambda$ \\
\hline \multirow{2}{*}{$(30,10)$} & MEs & 0.0842 & 0.0432 & 1.3542 & 0.0846 & 0.0435 & 1.3539 & 0.0921 & 0.0489 & 1.2874 \\
\hline & MSEs & 0.0345 & 0.0142 & 0.2114 & 0.0341 & 0.0140 & 0.2111 & 0.0275 & 0.0095 & 0.1874 \\
\hline \multirow{2}{*}{$(30,20)$} & MEs & 0.0882 & 0.0481 & 1.3498 & 0.0890 & 0.0495 & 1.3482 & 0.0981 & 0.0482 & 1.2811 \\
\hline & MSEs & 0.0318 & 0.0123 & 0.2089 & 0.0315 & 0.0119 & 0.2090 & 0.0254 & 0.0074 & 0.1836 \\
\hline \multirow{2}{*}{$(30,30)$} & MEs & 0.0921 & 0.0491 & 1.3450 & 0.0911 & 0.0521 & 1.344 & 0.0992 & 0.0482 & 1.2711 \\
\hline & MSEs & 0.0287 & 0.0100 & 0.2042 & 0.0277 & 0.0098 & 0.2032 & 0.0211 & 0.0069 & 0.1801 \\
\hline \multirow{2}{*}{$(50,15)$} & MEs & 0.0874 & 0.0471 & 1.3511 & 0.0880 & 0.0482 & 1.3490 & 0.0994 & 0.0487 & 1.2815 \\
\hline & MSEs & 0.0325 & 0.0134 & 0.2096 & 0.0324 & 0.0131 & 0.2097 & 0.0262 & 0.0081 & 0.1841 \\
\hline \multirow{2}{*}{$(50,30)$} & MEs & 0.0924 & 0.0493 & 1.3455 & 0.0909 & 0.0519 & 1.3510 & 0.0990 & 0.0487 & 1.2707 \\
\hline & MSEs & 0.0284 & 0.0103 & 0.2046 & 0.0273 & 0.0095 & 0.2037 & 0.0215 & 0.0071 & 0.1804 \\
\hline \multirow{2}{*}{$(50,50)$} & MEs & 0.1201 & 0.0511 & 1.3222 & 0.1217 & 0.0509 & 1.3001 & 0.0997 & 0.0503 & 1.2110 \\
\hline & MSEs & 0.0249 & 0.0074 & 0.2001 & 0.0242 & 0.0064 & 0.1987 & 0.0182 & 0.0050 & 0.1641 \\
\hline \multirow{2}{*}{$(70,35)$} & MEs & 0.0960 & 0.0499 & 1.3441 & 0.0939 & 0.0511 & 1.3451 & 0.0998 & 0.0497 & 1.2670 \\
\hline & MSEs & 0.0271 & 0.0098 & 0.2033 & 0.0265 & 0.0087 & 0.2026 & 0.0209 & 0.0063 & 0.1795 \\
\hline \multirow{2}{*}{$(70,50)$} & MEs & 0.1150 & 0.0508 & 1.3200 & 0.1207 & 0.0480 & 1.2900 & 0.0994 & 0.0509 & 1.2121 \\
\hline & MSEs & 0.0244 & 0.0071 & 0.2004 & 0.0239 & 0.0056 & 0.1981 & 0.0176 & 0.0047 & 0.1640 \\
\hline \multirow{2}{*}{$(70,70)$} & MEs & 0.1070 & 0.0503 & 1.1410 & 0.1002 & 0.0510 & 1.1591 & 0.1070 & 0.0503 & 1.1100 \\
\hline & MSEs & 0.0201 & 0.0025 & 0.1751 & 0.0185 & 0.0023 & 0.1690 & 0.0121 & 0.0011 & 0.1574 \\
\hline
\end{tabular}

Table 7: MILs and CPs from $\omega=\{0.1,0.05,1.0\}$.

\begin{tabular}{|c|c|c|c|c|c|c|c|c|c|c|}
\hline \multirow{2}{*}{$(n, m)$} & \multirow{2}{*}{ Tolls } & \multicolumn{3}{|c|}{ MLE } & \multicolumn{3}{|c|}{ Bayes $\left(P_{0}\right)$} & \multicolumn{3}{|c|}{ Bayes $\left(P_{1}\right)$} \\
\hline & & $\alpha$ & $\beta$ & $\lambda$ & $\alpha$ & $\beta$ & $\lambda$ & $\alpha$ & $\beta$ & $\lambda$ \\
\hline \multirow{2}{*}{$(30,10)$} & MILs & 0.2342 & 0.1234 & 1.7241 & 0.2329 & 0.1223 & 1.7219 & 0.2329 & 0.1223 & 1.7219 \\
\hline & CPs & $(0.88)$ & $(0.90)$ & $(0.88)$ & $(0.91)$ & $(0.89)$ & $(0.90)$ & $(0.92)$ & $(0.90)$ & $(0.91)$ \\
\hline \multirow{2}{*}{$(30,20)$} & MILs & 0.2323 & 0.1215 & 1.7219 & 0.2311 & 0.1204 & 1.7202 & 0.2311 & 0.1201 & 1.7200 \\
\hline & CPs & $(0.89)$ & $(0.91)$ & $(0.90)$ & $(0.91)$ & $(0.91)$ & $(0.92)$ & $(0.92)$ & $(0.91)$ & $(0.93)$ \\
\hline \multirow{2}{*}{$(30,30)$} & MILs & 0.2280 & 0.1191 & 1.7171 & 0.2285 & 0.1172 & 1.7169 & 0.2293 & 0.1177 & 1.7168 \\
\hline & CPs & $(0.92)$ & $(0.91)$ & $(0.92)$ & $(0.93)$ & $(0.94)$ & $(0.93)$ & $(0.91)$ & $(0.93)$ & $(0.91)$ \\
\hline \multirow{2}{*}{$(50,15)$} & MILs & 0.2327 & 0.1218 & 1.7223 & 0.2317 & 0.1211 & 1.7208 & 0.2319 & 0.1207 & 1.7206 \\
\hline & CPs & $(0.91)$ & $(0.90)$ & $(0.92)$ & $(0.93)$ & $(0.91)$ & $(0.94)$ & $(0.93)$ & $(0.92)$ & $(0.92)$ \\
\hline \multirow{2}{*}{$(50,30)$} & MILs & 0.2277 & 0.1190 & 1.7168 & 0.2282 & 0.1175 & 1.7171 & 0.2290 & 0.1174 & 1.7163 \\
\hline & CPs & $(0.92)$ & $(0.92)$ & $(0.91)$ & $(0.94)$ & $(0.91)$ & $(0.93)$ & $(0.92)$ & $(0.94)$ & $(0.92)$ \\
\hline \multirow{2}{*}{$(50,50)$} & MILs & 0.2224 & 0.1163 & 1.7132 & 0.2227 & 0.1161 & 1.7125 & 0.2239 & 0.1126 & 1.7125 \\
\hline & CPs & $(0.93)$ & $(0.93)$ & $(0.92)$ & $(0.92)$ & $(0.93)$ & $(0.94)$ & $(0.97)$ & $(0.92)$ & $(0.94)$ \\
\hline \multirow{2}{*}{$(70,35)$} & MILs & 0.2268 & 0.1181 & 1.7149 & 0.2275 & 0.1170 & 1.7163 & 0.2281 & 0.1167 & 1.7157 \\
\hline & CPs & $(0.91)$ & $(0.92)$ & $(0.92)$ & $(0.92)$ & $(0.93)$ & $(0.92)$ & $(0.93)$ & $(0.92)$ & $(0.93)$ \\
\hline \multirow{2}{*}{$(70,50)$} & MILs & 0.2227 & 0.1161 & 1.7129 & 0.2225 & 0.1158 & 1.7126 & 0.2241 & 0.1123 & 1.7122 \\
\hline & CPs & $(0.93)$ & $(0.94)$ & $(0.95)$ & $(0.94)$ & $(0.94)$ & $(0.94)$ & $(0.95)$ & $(0.94)$ & $(0.95)$ \\
\hline \multirow{2}{*}{$(70,70)$} & MILs & 0.2201 & 0.1143 & 1.7114 & 0.2199 & 0.1142 & 1.7109 & 0.2225 & 0.1111 & 1.7104 \\
\hline & CPs & $(0.92)$ & $(0.91)$ & $(0.95)$ & $(0.94)$ & $(0.92)$ & $(0.95)$ & $(0.92)$ & $(0.92)$ & $(0.93)$ \\
\hline
\end{tabular}




\section{Conclusion}

Different types of censoring schemes are available, see [27], and the type-II censoring scheme presents the simple type of censoring, saving the number of failures needed for statistical inference. Also, simulation studies are constructed to check the validity of the developed theoretical result, see [28, 29]. The problem of "time-to-failure" under different causes of failure is common in reliability studying. In natural, causes of failure may be dependent but in our modeling, we proposed that the causes of failure are independent. This model considers that the unit failure time is distributed with NRMW distribution with type-II censoring scheme. In this section, the simulation results have reported some points described as follows.

(1) The proposed model under the type-II censoring scheme for competing risks' model serves well for all choice of censoring schemes and parameters' choices

(2) The Bayes estimation under noninformative prior is more closed to maximum likelihood estimation

(3) The informative priors serve better than noninformative prior and maximum likelihood estimations

(4) By increasing the value of the affect sample size $m$, the values of the MSE and MIL reduce.

(5) The numerical results are more suitable when the proportion $(\mathrm{m} / \mathrm{n})$ is increasing.

\section{Data Availability}

No data were used to support the findings of this study.

\section{Conflicts of Interest}

The authors declare that they have no conflicts of interest regarding the publication of the paper.

\section{Acknowledgments}

This work was supported by Taif University under researchers supporting project number TURDP-2020/253, Taif, Saudi Arabia.

\section{References}

[1] S. Rajarshi and M. B. Rajarshi, "Bathtub distributions: a review," Communications in Statistics-Theory and Methods, vol. 17, no. 8, pp. 2597-2621, 1988.

[2] D. N. P. Murthy, M. Xie, and R. Jiang, Weibull Models, John Wiley and Sons, New York, NY, USA, 2003.

[3] H. Pham and C.-D. Lai, "On recent generalizations of the weibull distribution," IEEE Transactions on Reliability, vol. 56, no. 3, pp. 454-458, 2007.

[4] C. D. Lai, M. Xie, and D. N. P. Murthy, "A modified weibull distribution," IEEE Transactions on Reliability, vol. 52, no. 1, pp. 33-37, 2003.

[5] M. Bebbington, C.-D. Lai, and R. Zitikis, "A flexible weibull extension," Reliability Engineering \& System Safety, vol. 92, no. 6, pp. 719-726, 2007.
[6] M. Xie, Y. Tang, and T. N. Goh, "A modified Weibull extension with bathtub-shaped failure rate function," Reliability Engineering \& System Safety, vol. 76, no. 3, pp. 279-285, 2002.

[7] M. Xie and C. D. Lai, "Reliability analysis using an additive weibull model with bathtub-shaped failure rate function," Reliability Engineering and System Safety, vol. 52, pp. 87-93, 1995.

[8] A. M. Sarhan and J. Apaloo, "Exponentiated modified weibull extension distribution," Reliability Engineering \& System Safety, vol. 112, pp. 137-144, 2013.

[9] A. M. Sarhan, A. E.-B. A. Ahmad, and I. A. Alasbahi, "Exponentiated generalized linear exponential distribution," Applied Mathematical Modelling, vol. 37, no. 5, pp. 28382849, 2013.

[10] F. Famoye, C. Lee, and O. Olumolade, "The beta-weibull distribution," Journal of Statistical Theory and Applications, vol. 4, pp. 121-136, 2005.

[11] J. M. F. Carrasco, E. M. M. Ortega, and G. M. Cordeiro, “A generalized modified Weibull distribution for lifetime modeling," Computational Statistics \& Data Analysis, vol. 53, no. 2, pp. 450-462, 2008.

[12] S. J. Almalki and J. Yuan, "A new modified Weibull distribution," Reliability Engineering \& System Safety, vol. 111, pp. 164-170, 2013.

[13] S. J. Almalki, "A reduced new modified Weibull distribution," Journal of Communications in Statistics-Theory and Methods, vol. 47, pp. 2297-2313, 2018.

[14] D. R. Cox, "The analysis of exponentially distributed life-times with two types of failure," Journal of the Royal Statistical Society: Series B (Methodological), vol. 21, no. 2, pp. 411-421, 1959.

[15] H. A. David and M. L. Moeschberger, The Theory of Competing Risks, Griffin, London, UK, 1978.

[16] M. J. Crowder, Classical Competing Risks, Chapman and Hall, London, UK, 2001.

[17] N. Balakrishnan and D. Han, "Exact inference for a simple step-stress model with competing risks for failure from exponential distribution under type-II censoring," Journal of Statistical Planning and Inference, vol. 138, pp. 4172-4186, 2008.

[18] A. Ganguly and D. Kundu, "Analysis of simple step-stress model in presence of competing risks," Journal of Statistical Computation and Simulation, vol. 86, no. 10, pp. 1989-2006, 2016.

[19] A. Ali, A. M. Almarashi, and G. A. Abd-Elmougod, "Statistical analysis of competing risks lifetime data from Nadarajaha and haghighi distribution under type-II censoring," Journal of Intelligent and Fuzzy Systems, vol. 38, pp. 2591-2601, 2019.

[20] A. M. Almarashi and G. A. Abd-Elmougod, "Accelerated competing risks model from Gompertz lifetime distributions with type-II censoring scheme," Thermal Science, vol. 24, pp. 165-175, 2019.

[21] N. Metropolis, A. W. Rosenbluth, M. N. Rosenbluth, A. Teller, and E. Teller, "Equation of state calculations by fast computing machines," The Journal of Chemical Physics, vol. 21, no. 6, pp. 1087-1092, 1953.

[22] A. Ali, A. M. Almarashi, and G. A. Abd-Elmougod, "Joint type-I generalized hybrid censoring for estimation the two Weibull distributions," Journal of Information Science and Engineering, vol. 36, pp. 1243-1260, 2020.

[23] D. G. Hoel, "A representation of mortality data by competing risks," Biometrics, vol. 28, no. 2, pp. 475-488, 1972.

[24] B. Pareek, D. Kundu, and S. Kumar, "On progressively censored competing risks data for weibull distributions," 
Computational Statistics \& Data Analysis, vol. 53, no. 12, pp. 4083-4094, 2009.

[25] A. M. Sarhan, D. C. Hamilton, and B. Smith, "Statistical analysis of competing risks models," Reliability Engineering \& System Safety, vol. 95, no. 9, pp. 953-962, 2010.

[26] E. Cramer and A. B. Schmiedt, "Progressively type-II censored competing risks data from lomax distributions," Computational Statistics \& Data Analysis, vol. 55, no. 3, pp. 1285-1303, 2011.

[27] N. Balakrishnan and R. Aggarwala, Progressive Censoring-Theory, Methods, and Applications, Springer Science+ Business Media, Berlin, Germany, 2000.

[28] A. A. Soliman, A. H. Abd Ellah, N. A. Abou-Elheggag, and G. A. Abd-Elmougod, "A simulation-based approach to the study of coefficient of variation of Gompertz distribution under progressive first-failure censoring," Indian Journal of Pure and Applied Mathematics, vol. 42, no. 5, pp. 335-356, 2011.

[29] A. A. Soliman, A. H. Abd-Ellah, N. A. Abou-Elheggag, and G. A. Abd-Elmougod, "Estimation of the parameters of life for Gompertz distribution using progressive first-failure censored data," Computational Statistics \& Data Analysis, vol. 56, no. 8, pp. 2471-2485, 2012. 